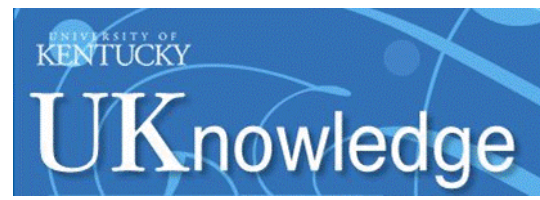

University of Kentucky

UKnowledge

\title{
A Randomized Trial of Brief Assessment Interventions for Young Adults Who Use Drugs in the Club Scene
}

\author{
Steven P. Kurtz \\ Nova Southeastern University \\ Mance E. Buttram \\ Nova Southeastern University \\ Maria E. Pagano \\ Case Western Reserve University \\ Hilary L. Surratt \\ University of Kentucky, hilary.surratt@uky.edu
}

Follow this and additional works at: https://uknowledge.uky.edu/chsr_facpub

Part of the Behavior and Behavior Mechanisms Commons, Health Services Research Commons, and the Substance Abuse and Addiction Commons

Right click to open a feedback form in a new tab to let us know how this document benefits you.

\section{Repository Citation}

Kurtz, Steven P.; Buttram, Mance E.; Pagano, Maria E.; and Surratt, Hilary L., "A Randomized Trial of Brief Assessment Interventions for Young Adults Who Use Drugs in the Club Scene" (2017). Center for Health Services Research Faculty Publications. 5.

https://uknowledge.uky.edu/chsr_facpub/5

This Article is brought to you for free and open access by the Center for Health Services Research at UKnowledge. It has been accepted for inclusion in Center for Health Services Research Faculty Publications by an authorized administrator of UKnowledge. For more information, please contact UKnowledge@lsv.uky.edu. 


\section{A Randomized Trial of Brief Assessment Interventions for Young Adults Who Use Drugs in the Club Scene}

\section{Digital Object Identifier (DOI)}

https://doi.org/10.1016/j.jsat.2017.05.008

\section{Notes/Citation Information}

Published in Journal of Substance Abuse Treatment, v. 78, p. 64-73.

(C) 2017 Elsevier Inc. All rights reserved.

This manuscript version is made available under the CC-BY-NC-ND 4.0 license https://creativecommons.org/licenses/by-nc-nd/4.0/.

The document available for download is the author's post-peer-review final draft of the article. 
J Subst Abuse Treat. 2017 July ; 78: 64-73. doi:10.1016/j.jsat.2017.05.008.

\title{
A Randomized Trial of Brief Assessment Interventions for Young Adults who Use Drugs in the Club Scene
}

\author{
Steven P. Kurtz ${ }^{a}$, Mance E. Buttram ${ }^{a}$, Maria E. Pagano ${ }^{b}$, and Hilary L. Surratt ${ }^{c}$ \\ ${ }^{a}$ Center for Applied Research on Substance Use and Health Disparities, Nova Southeastern \\ University, 7255 NE $4^{\text {th }}$ Avenue, Suite 112, Miami, FL 33138 USA \\ bDepartment of Psychiatry, Division of Child Psychiatry, Case Western Reserve University, 10524 \\ Euclid Avenue \#1155A, Cleveland, OH 44106 USA \\ 'Center for Health Services Research, Department of Internal Medicine, University of Kentucky, \\ 740 South Limestone Avenue, Lexington, Kentucky 40536
}

\begin{abstract}
Background-Efficacious interventions to reduce drug use and its consequences for club drug using populations are not apparent in the literature. We tested interviewer-(CAPI) and selfadministered (ACASI) comprehensive health and social risk assessments as distinct interventions compared to waitlist control.
\end{abstract}

Methods-750 men and women ages 18-39 with multidrug use and heterosexual behavior were randomized in equal proportions to the three conditions. Instrumentation included well-tested measures of drug use, risky sex, mental distress and substance dependence.

Results-The sample was 56\% male; mean age $=25$. Reported risk behaviors and health consequences did not differ by assessment modality. Adjusted HLM analyses showed a significant main effect of assigned condition on all outcomes. CAPI participants had greater reductions in drug use, risky sex, mental distress and substance dependence symptoms, and greater increases in abstinence, compared to ACASI intervention or control participants at 12 months, except that the CAPI and ACASI conditions had similar efficacy for reductions in drug use. Effect sizes for CAPI versus ACASI participants were $d=0.2-0.3$, and between CAPI and controls $d=0.2-0.4$. Effect sizes for improved outcomes between ACASI compared to controls were small to non-significant.

Conclusions-The study established the therapeutic benefit of interviewer interaction in reducing risky behavior among this young drug using population. The study demonstrated the efficacy and acceptability of a low threshold intervention in reducing drug use, sexual risk and related co-morbidities among a not-in-treatment young adult population that exhibits severe and complex levels of drug use, but that is also highly resistant to intervention.

Corresponding Author: Steven P. Kurtz, Ph.D., Center for Applied Research on Substance Use and Health Disparities, Nova Southeastern University, 7255 NE $4^{\text {th }}$ Avenue, Suite 112, Miami, FL 33138 USA, steven.kurtz@ nova.edu, Tel: (305) 571-2774. Conflicts of interest: None.

Publisher's Disclaimer: This is a PDF file of an unedited manuscript that has been accepted for publication. As a service to our customers we are providing this early version of the manuscript. The manuscript will undergo copyediting, typesetting, and review of the resulting proof before it is published in its final citable form. Please note that during the production process errors may be discovered which could affect the content, and all legal disclaimers that apply to the journal pertain. 


\section{Keywords}

young adult; club drugs; drug dependence; brief interventions

\section{Introduction}

Young adults who predominate in electronic dance music (EDM) and other nightclub subcultures are especially vulnerable to mixing numerous drugs during typical binges on weekends and at special events (Kurtz, Inciardi, Surratt, \& Cottler, 2005; Kurtz, Surratt, Buttram, Levi-Minzi, \& Chen, 2013; Owen, 2003; Silcott, 1999). The term "club drugs" traditionally refers to illicit stimulant and hallucinogenic drugs commonly used to enhance the club experience, including MDMA, powder cocaine, LSD, ketamine and GHB (National Institute on Drug Abuse, 2009; Parsons, Grov, \& Kelly, 2009; Reynolds, 1998), but these have expanded over the years to include psychoactive prescription medications (Kelly \& Parsons, 2007; Kelly, Wells, Pawson, LeClair, \& Parsons, 2014; Kurtz et al., 2005; Kurtz, Surratt, Buttram, Levi-Minzi, \& Chen, 2013), and an ever-evolving range of synthetic psychedelic and stimulant "designer" drugs (German, Fleckenstein, \& Hanson, 2014; Musselman \& Hampton, 2014).

The intensity and complexity of club-goers' substance use puts them at high risk for numerous health and social problems, including: tolerance, dependence, overdose, and chronic psychiatric symptoms, (Cottler, Womack, Compton, \& Ben-Abdallah, 2001; Kurtz et al., 2013; Kurtz, Surratt, Levi-Minzi, \& Mooss, 2011; Nicholi, 1983; Shifano, Di Furia, Gorza, Minicuci, \& Bricolo, 1998; Parrott, Milani, Parmar, \& Turner, 2001); difficulties with peer and other social relationships (Chinet, Stephan, Zobel, \& Halfon, 2007; Singer, Linares, Ntiri, Henry, \& Minnes, 2004); drunk/drugged driving (Voas, Johnson, \& Miller, 2013), criminal activity and violent victimization (Kurtz, 2012; Kurtz, Inciardi, \& Pujals, 2009); sexual risk behaviors (Buttram \& Kurtz, 2015a; Buttram \& Kurtz, 2016; Novoa, Ompad, Wu, Vlahov, \& Galea, 2005; Sterk, Klein, \& Elifson, 2008) and higher rates of sexually transmitted infections (STI; Mitcheson, McCambridge, Byrne, Hunt, \& Winstock, 2008).

The wide-ranging adverse consequences of substance use among this young population require intervention approaches that are feasible to implement, acceptable to the population, and effective in producing behavioral change. However, the use of euphoric and hallucinogenic drugs is widely viewed in the club culture as fun and fashionable, and few recognize the problematic aspects of substance use (Kurtz, et al., 2013; Whittingham et al., 2009; Abdulrahim \& Bowden-Jones, 2015). As a result, there is pervasive resistance to participation in formal interventions. Moreover, club scene participants tend to be suspicious of or disinterested in drug use and sexual risk prevention messages delivered by governmental or health authorities; peers and internet sites are more trusted sources of this information (Carlson, Falck, McCaughan, \& Siegal, 2004; Falck, Carlson, Wang \& Siegal, 2004).

Few controlled intervention studies for club drug using populations are apparent in the literature. Researchers have found mixed success with the dissemination of educational materials (Whittingham et al., 2009), although one study of peer-delivered health messages 
showed promise (Sillins, Bleeker, Simpson, Dillon, \& Copeland, 2013). Two large trials comparing motivational enhancement therapy to educational campaigns found moderate post-intervention reductions in ecstasy use in both randomized conditions (Marsden et al., 2006; Norberg et al., 2014). Brief screening and referral interventions, while effective for alcohol use, have not demonstrated much success with diverse samples of drug users (Saitz 2014; White et al., 2015; Young et al., 2014).

To address this gap, we tested the efficacy of two brief assessment intervention conditions compared to a waitlist control in reducing drug and sex-related risk behavior among young adults with multidrug use in the EDM nightclub culture. This intervention approach emerged from an earlier natural history study of young men and women in the club scene with complex levels of drug use, which found that participation in periodic intervieweradministered comprehensive health and social risk assessments induced participants to sharply reduce their substance use and sexual risk behaviors over 18 months (Kurtz et al., 2013). Similar reactive effects of research study and/or clinical assessments, attributed to consciousness raising, self-monitoring, increased self-awareness, and/or self-efficacy, have been recognized since at least the mid-1970s (Clifford \& Maisto, 2000; Epstein et al., 2005; Lightfoot, Comulada, \& Stover, 2007). In fact, Clifford, Maisto, \& Davis (2007) noted the similarities of such assessments to formal substance abuse treatment, as both combine problem evaluation with perceived professional interest in one's well-being.

Given the robust findings of the natural history study, and the challenges in designing interventions for the population noted earlier, evaluating a potentially scalable, singlesession assessment-based intervention approach in a controlled trial appeared particularly promising. At the same time, computerized, self-administered interventions (not necessarily assessment-based) have emerged as useful tools for psychotherapy (Newman, 2004); treating insomnia (Schaffer, 2009), depression (McKendree-Smith, Floyd, \& Scogin, 2003), and cannabis use disorder (Budney et al. 2015); and reducing sexual risk behaviors and STI incidence (Noar, Black, \& Pierce, 2009), though there is mixed evidence regarding their effectiveness compared to clinician-delivered treatments (Greist et al., 2002 [clinician superior]; Budney et al., 2015 [clinican-computer equal]). Thus, we tested interviewer- and self-administered modes of assessment as distinct interventions to examine potential differences in their efficacy. This approach essentially tests the value added by the relational interview interaction in producing behavioral change, which aligns with a social influences perspective (Bandura, 1986).

\section{Methods}

\subsection{Study design}

The three-armed randomized controlled trial was designed to recruit a sample of 750 participants in order to achieve 80 percent power to detect 0.2 effect size reductions in primary outcomes - past 90-day substance use and condomless anal/vaginal sex frequencies - within intervention condition, and 0.3 effect size differences between conditions, assuming $20 \%$ attrition over the 12 month follow-up period. Drug/heavy alcohol abstinence, mental distress, and drug dependence symptoms were specified as secondary outcomes. The primary hypothesis was that participants assigned to the computer-assisted personal 
interview (CAPI) and audio computer-assisted self-interview (ACASI) assessment intervention conditions would reduce their sexual risks and substance use to a similar degree and to a significantly greater extent than those assigned to a waitlist control condition across all outcome measures. The protocol is registered with ClinicalTrials.gov \#NCT01362634.

\subsection{Site}

Miami-Dade County, Florida is a diverse community of 2.6 million people, of whom $66.2 \%$ are Hispanic, 18.9\% Black and 14.8\% White (U.S. Census Bureau, 2015). Specific to the EDM nightclub scene, Miami is an international destination for partying, sexual tourism, and club drug use - setting trends that are emulated and replicated globally (Brandt, 2003; Perrone, 2009).

\subsection{Sampling plan}

Participants (N=750) were recruited between September 2011 and November 2014 through respondent-driven sampling (RDS; Heckathorn, 1997). Seeds (initial respondents) were recruited through outreach at local nightclubs and existing contacts in the club culture. Each seed and subsequent study participant was provided with recruitment coupons to give to other drug users in their social network, with the understanding that they would earn $\$ 50$ for the recruitment of each additional eligible respondent. Based upon recruitment patterns in the prior natural history study, steering incentives (Heckathorn et al., 2002) of an additional $\$ 10$ were implemented to reward the recruitment of women and African Americans. Each participant-recruiter was limited to five coupons to prevent a few participants with large social networks from biasing the overall sample toward those with similar demographic and drug using profiles.

Inclusion criteria were: 1) ages 18-39; 2) heterosexual vaginal and/or anal sex in the past 90 days; 3 ) use of club drug(s), defined as powder cocaine, MDMA, LSD, methamphetamine, GHB and/or ketamine, at least three times in the past 90 days; 4) non-medical use of a psychoactive prescription medication in the past 90 days; 5) attendance at large local nightclubs at least once per month; 6) residence in metropolitan Miami with no plans to move away in the next year; and 7) willingness to provide a mailing address, personal telephone number, and email address or social media profile.

Eligibility was restricted to men and women who reported recent heterosexual behavior because the sexual risk reduction component of the intervention would need to be carefully targeted to be meaningful; men and women who also reported same-gender sex were not excluded. Substance use criteria were selected to match those in the earlier natural history study, and to ensure that drug use was sufficiently frequent and complex as to indicate the need for intervention. Pregnant women and anyone currently enrolled in a substance abuse treatment program were excluded. Follow-up interviews were completed in November 2015.

\subsection{Staffing}

Field staff were age-peers of the target population with at least a Bachelor's degree, one year of research experience, and an expressed commitment to substance abuse research. They were trained in the study recruitment procedures, human subjects protections, data collection 
instruments, intervention protocols, and completed requirements for State of Florida certification as HIV test counselors.

\subsection{Procedures}

The study was conducted at a field office located in a standard business office building near the center of the primary transportation routes of the County. Potential enrollees responding to recruitment messages or coupons were screened for eligibility by telephone. Screening instruments included queries about pseudo drugs and other non-eligibility measures to prevent dissemination of the actual criteria. Those who were eligible and interested in participating were scheduled for enrollment at the field office. The enrollment visit included five elements: 1) eligibility rescreening; 2) written informed consent, including a description of the study aims and the three intervention conditions, using procedures approved by the university's institutional review board; 3) collection of locator information; 4) HIV brief counseling and rapid test administration (721 tested, 23 refused, 6 self-reported HIV+ status); and 5) training in the RDS recruitment procedures. Enrollees were paid $\$ 30$ for their time and travel expenses, and scheduled for the baseline assessment and intervention visit within the next 1-2 weeks.

Enrollees who did not return for the baseline visit were replaced. At baseline, all participants completed a brief risk behavior inventory (RBI; 17 substance use frequency items and 8 sexual behavior items) assessing primary outcomes using an ACASI-based instrument that was designed to minimize reactive effects and took a median of 12 minutes to complete. Participants were randomized immediately thereafter in 1:1:1 ratio to the three study arms: 1) an interviewer-administered comprehensive health and social risk assessment intervention (CAPI Intervention); 2) a self-administered assessment intervention with identical instrumentation (ACASI Intervention); and 3) a waitlist control condition (Control). Randomization was based on a computer-generated random number table specified by the total sample size and equal probability of assignment to the three conditions. Sequentiallynumbered opaque envelopes containing the arm assignment designations were prepared in the research office and delivered in batches to the field office director. Field staff requested the next envelope in sequence following each participant's completion of the baseline RBI; the participant opened the envelope in the presence of the staff member. All participants received free condoms and a $\$ 50$ stipend upon completion of these baseline activities, which lasted approximately 30 minutes. During this same visit and immediately after randomization, those assigned to the CAPI or ACASI arms continued with the intervention protocols (see Interventions, below).

\subsection{Interventions}

The comprehensive health and social risk assessment interventions, which may be found at http://arsh.nova.edu/publications/forms/intervention-instrumentation.pdf, included identical instrumentation for CAPI and ACASI administration, and were programmed using the QDS ${ }^{\text {TM }}$ Questionnaire Development System (Nova Research 2009). The programming included clear skip patterns, well-defined value ranges to bound answer selection, checks for consistency with answers to earlier questions on the same topic, and required a response 
entry (including "don't know" and "refuse to answer" choices) before moving on to the next question.

The CAPI intervention was administered in a private office by a trained interviewer who read the questions verbatim from the QDS ${ }^{\mathrm{TM}}$ screens to the participant and recorded their responses on a laptop computer. Research assistants were trained to hear and record participants' responses to assessment items without signaling judgment, discomfort, surprise or emotion, as well as avoiding extraneous conversation or deviating from the interview instrument. The ACASI intervention was loaded onto one of several desktop computers that were housed in private cubicles; participants' read and/or listened to the questions and recorded their own responses.

The interventions were based upon the assessments used for the natural history study, but enhanced in several areas to increase participants' critical awareness of the health and social risks posed by substance use and condomless sex. The core instrumentation was the Global Appraisal of Individual Needs (GAIN, v. 5.4; Dennis, 2006), which has eight core sections (background, substance use, physical health, sexual risk behaviors, mental health, victimization, criminal justice involvement, and education/work/financial stability), with each containing questions on recency of problems, breadth of symptoms, and recent (90 day) and lifetime prevalence in days or times. The items include scales for Diagnostic and Statistical Manual of Mental Disorders, $4^{\text {th }}$ edition (DSM-IV)-based diagnoses (American Psychiatric Association, 1994). Psychometric studies have found Cronbach's alphas between .8 and .9. Behavior questions have demonstrated test-retest correlations over 8 (Dennis, Rourke, Lennox, Campbell \& Caddell, 1995).

For this study, the list of drugs was extended to include major classes of psychoactive prescription medications. The sexual behavior questions were expanded to include counts of total and condomless vaginal and anal sex events; number, gender and types of sex partners (primary vs. casual); substances used before or during sex; agreements and knowledge about monogamy in primary relationships; group sex behaviors; and knowledge of sex partners' HIV status. We also enhanced the assessment to include items focused on friendship characteristics and social support; social acceptability of various drug use and sexual behaviors; resilience, coping and distress tolerance; goal-setting and achievement; social activities and community involvement; and readiness to change substance use and sexual behaviors.

The baseline assessments (as well as the 12 month assessments for control arm participants) took a median of 76 minutes for ACASI participants and 89 minutes for CAPI participants to complete. The 12 month assessments for the intervention groups excluded life history items collected at baseline and took a median of 68 minutes to complete.

Fidelity monitoring. CAPI interviews were assigned to interviewers on the basis of schedule availability without regard to participant characteristics. All CAPI interventions were audiorecorded for auditing and training. The first 5 interviews conducted by each interviewer were audited by non-project research staff who listened to the audio recording, re-keyed the interview to identify any errors, and noted any deviations from protocol. Following these 
initial audits, two interviews per interviewer per month were audited to maintain the fidelity of the CAPI intervention. No baseline differences in outcome measures by interviewer were observed. ACASI assessments were examined by field staff for completeness and consistency of responses before the participant left the field office to allow participants to complete any unintentionally missed items and to clarify discrepant responses.

\subsection{Follow-Up visits}

At 3-, 6- and 12-months post-baseline, all participants again completed the ACASI-based RBI that measured primary outcome data. At 12-month follow-up, those assigned to the control condition received the CAPI assessment intervention, while those assigned to the CAPI and ACASI intervention conditions completed an interviewer-administered instrument that was similar to the intervention assessment, inclusive of secondary outcome data but exclusive of life history items. Using this design, data collection procedures were consistent at each wave and across arms, with the only difference that those assigned to the two intervention groups completed the CAPI or ACASI comprehensive health and social risk assessment at baseline. Respondents received $\$ 50$ for their time and travel costs for each visit.

\subsection{Measures}

Demographic measures included age, gender, race/ethnicity, and education. Race/ethnicity was assessed by asking participants if they were Hispanic or Latino, followed by asking what race they considered themselves to be. Education was assessed based on high school completion vs. not.

Substance use measures included past 90-day frequencies, in days ("in the past 90 days, since [anchor point], how many days have you used any ...”), of use of alcohol, marijuana, powder cocaine, ecstasy, LSD, other hallucinogens (e.g., psilocybin, PCP; ketamine; 2cx, DMT), methamphetamine, and heroin, as well as the non-medical use of prescription benzodiazepines, opioids, stimulants, antidepressants and antipsychotics. For descriptive reporting, baseline substance use measures were dichotomized into "yes" and "no". For longitudinal analyses of substance use outcomes, we constructed a past 90-day composite club drug use measure equal to the sum of days of use of the four most prevalent club drugs: powder cocaine, ecstasy, and prescription benzodiazepines and opioids. The instrumentation also included a global measure of drug/heavy alcohol abstinence that was used in the efficacy analyses, "During the past 90 days, on how many days did you go without using 5 or more drinks, marijuana, cocaine, or any other drug?"

Sexual behavior questions included counts of past 90-day sex partners and condomless vaginal and anal intercourse events. To assess potential infectious disease exposure, risky vaginal and/or anal sex was defined as condomless vaginal and/or anal intercourse in the context of a non-monogamous relationship or with a non-primary partner during the past 90 days. For descriptive reporting, baseline risky sex measures were dichotomized into "yes" and "no".

The GAIN includes the General Mental Distress Scale (GMDS), which includes past year DSM-IV symptom counts of depression ( 9 items), anxiety (12 items), and somatic disorders 
(four items). Alpha reliability coefficients for the depression, anxiety, and somaticism subscales in this study were $0.882,0.887$, and 0.801 , respectively. The GMDS is further reducible to classifications indicating clinical significance - subclinical, moderate, and severe (Dennis, 2006) - and was dichotomized into "severe" (seven or more symptoms) and "not severe" for descriptive purposes. Substance dependence was assessed by seven DSMIV symptoms during the past year (e.g., needing more drug to get the same effect; being unable to quit or cut down), and was dichotomized as "dependent" (three or more symptoms) or "not dependent" for descriptive reporting.

Victimization was assessed by affirmative responses to any of the following events: being attacked with a weapon or being beaten so as to cause bruises, cuts or broken bones (physical abuse); being forced to participate in sexual acts against one's will (sexual abuse); or being made to feel very bad about oneself or one's life (emotional abuse). Age of first instance of any type of abuse was dichotomized into "victimized before the age of 18 " vs. not.

\subsection{Data Analyses}

Statistical analyses were performed using the procedures CORR, FREQ, MIXED, and GLIMMIX of SAS Version 9.3 (SAS Institute Inc., Cary, NC). There were no missing data on completed assessments, except for one ACASI participant who refused secondary outcome items and age of first victimization, and six ACASI participants who responded "don't know" to extent of recent abstinence. For the baseline to 3 month outcomes, there were 684 cases available for analysis; for baseline to 6 months, 669 cases; and for baseline to 12 months, 602 cases. All outcomes were examined on an intent-to-treat basis, and included all data available at the four study intervals. There were no differences in baseline characteristics or arm assignment between cases with $(\mathrm{N}=602)$ and without $(\mathrm{N}=148)$ complete follow-up data, and missing data patterns were nonmonotone.

Means, standard deviations, and frequencies were calculated for descriptive reporting. Except for substance dependence symptoms, distributions of outcome measures were positively skewed and required logarithmic transformations for longitudinal analyses. In accordance with the Consolidated Standards of Reporting Trials (CONSORT; Schultz, Altman, \& Moher, 2010) statement, we report a summary of unadjusted outcomes by arm and study interval. Effect size estimates of individual-level change (unadjusted) were calculated from the mean difference between baseline and 12 month scores divided by the standard deviation of the change score, corrected by the correlation between time points (i.e., [(M1 - M2) / SD] / $(1$ - r); Cohen, 1988). Statistics reported are based on logtransformed measures, including Cohen's $d$ effect size statistics and related 95\% confidence intervals. Formulas for converting between $F$, eta ${ }^{2}$, and $d$ were taken from Cohen (1988). We characterize these $d$ statistics using Cohen's categories of 'small' ( $d=0.2)$, 'medium' ( $d=$ $0.5)$, and 'large' ( $d=0.8)$ (Cohen, 1988).

Five hierarchical linear models (HLMs) were constructed to evaluate the efficacy of the interventions on outcomes. Two primary (club drug use and risky sex frequencies) and three secondary (days abstinent from drug/heavy alcohol use; mental distress and substance dependence symptoms) outcomes were pre-specified in the trial protocol. In accordance with 
the study design, secondary outcomes at baseline were not collected among controls, as they did not complete a comprehensive assessment until 12 month follow-up. As pre-specified in the trial's protocol, we used the Markov chain Monte Carlo method for multiple imputations with weighted covariates (Little \& Rubin, 2002) for missing data reconstruction of the baseline values of secondary outcomes for controls. Weighted covariates included history of DUI, intoxication, and treatment episodes; and diagnostic history of anxiety, depression, and post-traumatic stress disorder. The statistics reported for these variables are the averaged results across 50 imputations. We note that baseline values of secondary outcome measures could not be estimated using these procedures for control arm participants who did not complete a 12-month assessment.

Risky anal/vaginal sex incidences were analyzed using Poisson regression methods recommended for count data with random intercepts by maximum likelihood and with a robust variance correction (Allison, 2005). Random effects included subjects and time with an unstructured correlation matrix to allow for all possible correlations between the random intercept, random slope, and time. Models predicting primary outcomes included a time term, a quadratic time term, and time*arm interaction term. Using the Tukey-Kramer adjustment for multiple comparisons in HLM models, HLM models included contrast statements to determine effect size differences between arm conditions. Parameter estimates were adjusted for characteristics prognostic of outcomes (age; Pagano, Zemore, Onder \& Stout, 2009) and gender and race/ethnicity subgroups in accordance with the CONSORT statement and NIH reporting requirements. When multicollinearity diagnostics of fitted models were examined, tolerances were .50 and above, indicating low concern of multicollinearity (Allison, 1999). The family-wise error rate for the two sets of outcomes was set at .05 (two tailed).

\section{Results}

\subsection{Sample characteristics}

Sample characteristics at baseline by intervention condition are shown in Table 1. On average, participants were 25.4 years of age $(S D=5.4$, range $=18-39)$ and the large majority (85.3\%) had completed high school. About half $(56.1 \%)$ were male; the racial/ethnic composition reflected Miami-Dade County's population: $65.7 \%$ Hispanic; $20.4 \%$ Black, $11.6 \%$ White and $2.3 \%$ other race/ethnicity. In the 90 days prior to baseline, large majorities ( $88 \%$ or more) endorsed the use of alcohol, marijuana, cocaine, MDMA, benzodiazepines and/or opioid analgesics; about half used LSD, other hallucinogens and/or prescription stimulants; and more than $20 \%$ endorsed methamphetamine, heroin, and prescription antipsychotics and/or antidepressants.

The sample had an average of $6.9(\mathrm{SD}=13.3$; median $=3)$ sex partners in the past 90 days; more than half had condomless vaginal sex, and more than one-quarter condomless anal sex, in non-monogamous relationships or with casual partners. About half (51\%) reported symptoms of severe mental distress and $68.8 \%$ met DSM-IV criteria for substance dependence. Three out of four (74.5\%) had histories of victimization, with $58.7 \%$ suffering abuse before the age of 18 . The intervention groups were not significantly different at baseline on demographics, substance use, sexual behaviors, or psychiatric comorbidities. 


\subsection{Enrollment, retention and adverse events}

The participant flow chart is shown in Figure I. The most common reasons for failing the initial eligibility screen $(\mathrm{N}=1326)$ were: drug use threshold (64.9\%); sexual behavior threshold (21.6\%); lack of intention to remain in the Miami area for 12 months (14.2\%); age (8.4\%); clubbing frequency threshold (6.9\%); and recent enrollment in a substance abuse treatment program $(3.5 \%)$, or some combination of these. Of the 750 men and women randomized into the study: 557 participants $(74.3 \%)$ completed all four assessments; 122 (16.3\%) completed baseline plus two follow-ups; 40 (5.3\%) completed baseline plus one follow-up; and 31 (4.1\%) completed only the baseline assessment. Participants missing one or more assessments did not differ from those with complete data in terms of age, gender, race/ethnicity, victimization history, or primary or secondary outcome measures at baseline. Attrition rates were similar across conditions.

Eleven participants withdrew from the study after baseline assessment for various reasons, including scheduling conflicts with employment or moving away from the area. Twenty participants were withdrawn from the study because they became members of vulnerable population groups for whom approvals for participation were not sought (i.e., pregnant women and prisoners). All other participants lost to follow-up were due to loss of contact with study staff. Although a number of participants experienced drug treatment admissions and medical problems over the course of the study, no adverse events were attributable to study participation. Two serious adverse events (deaths) occurred, but neither was related to study participation.

\subsection{Study outcomes}

Table 2 presents the means and standard deviations for study outcomes (unadjusted) by condition at each assessment point, together with the mean changes from baseline to 12 months and the corresponding effect sizes. Large effect sizes $(d=0.82-0.89)$ for the composite club drug use measure were found for both the CAPI and ACASI intervention conditions. Small to medium effect sizes $(d=0.20-0.54)$ were found for the primary sexual risk and the secondary outcome measures in both intervention conditions, except that only the CAPI condition showed a significant increase in the number of days abstinent. In contrast, the effect sizes for risk reduction in the control condition were medium for the primary composite drug use outcome, small for the primary sexual risk outcome, and nonsignificant for secondary outcomes. In terms of clinical significance, participants in the CAPI intervention reported reductions in substance dependence and mental distress symptoms, on average, to below important diagnostic thresholds ( 3 or more substance dependence and 7 or more mental distress symptoms). Participants in the ACASI arm reported reductions in mental distress and substance dependence symptoms to about the clinically-significant cut points for these comorbidities, and those in the control group experienced no abatement of symptoms.

The results showing the efficacy of the two brief assessment interventions compared to controls on primary and secondary outcomes are shown in Table 3. Controlling for prespecified model covariates, the HLM analyses show a significant main effect of arm on primary and secondary outcomes. CAPI participants showed greater reductions in club drug 
use and risky vaginal sex frequencies, and mental distress and substance dependence symptoms, and greater increases in days abstinent from drug/heavy alcohol use, compared to those assigned to the ACASI intervention or control conditions, except that the CAPI and ACASI conditions had similar efficacy for reduction in club drug use frequency. Effect sizes for the greater improvements among CAPI versus ACASI participants were small $(d=$ 0.2-0.3) and between CAPI and controls small to medium $(d=0.2-0.4)$. Effect sizes for improved outcomes in the ACASI arm compared to controls were small for club drug use frequency and non-significant for risky vaginal sex frequency. On secondary outcomes, ACASI outperformed the control condition by small $(d=0.2)$ effect size differences in days abstinence and substance dependence symptoms, but no difference was found for mental distress symptoms. Thus, we found support for our hypothesis of significantly greater reductions in substance use and sexual risk behaviors among participants assigned to either intervention in comparison to waitlist controls, but only partially, as the CAPI intervention was more efficacious than the ACASI modality across four of five measures.

Collection of primary outcome data at four assessment periods allowed for the examination of changes in those outcomes over time in the sample overall, changes in the rate of change over time (time ${ }^{2}$ ), and whether the rate of change differed by condition (i.e. time $\mathrm{X}$ arm interaction). For risky vaginal sex frequency, the rate of change was non-linear, with the greater change in the initial follow-up period, and was more persistent among intervention groups compared to controls. There were no main effects of age, gender, or race/ethnicity on baseline measures of study outcomes.

\section{Discussion}

\subsection{Efficacy of comprehensive assessments as interventions}

Our primary hypothesis was partially confirmed, as the HLM analyses showed the interviewer-administered assessment intervention to be superior to the control group by meaningful margins across all outcomes. Most importantly, participants in the CAPI intervention reported reductions in substance dependence and mental distress symptoms to below clinically significant levels. However, contrary to our hypothesis, the ACASI condition showed lower efficacy, with small to non-significant effects compared to the control condition.

The effect sizes for reductions in drug use frequency and substance dependence symptoms in the CAPI arm approximate those reported in the parent natural history study of the same population (Kurtz et al., 2013). Taken together, these studies provide firm empirical support for the beneficial intervention effects of interviewer-administered comprehensive health and social risk assessments for not-in-treatment young adult drug users. Although ACASI showed modest efficacy compared to the controls on some outcome measures, it appears that interviewer interaction accounts for the major difference in assessment effects.

Although the precise mechanism of action is unclear, the interpersonal interviewer interaction likely plays a role in improving participants' processing and retention of assessment items, as well as providing a backdrop of empathy, tolerance, and openness, and the opportunity for verbal reflection on assessment items. We believe that comparisons with 
computer- vs. professional therapist-delivered intervention trials of behavioral therapies (e.g., Budney et al., 2015) are not especially relevant to these findings. Our study targeted a sample resistant to intervention and was situated outside of a therapeutic setting. Participants were randomized to an experience of either self-recording their behaviors, attitudes and social experiences on a computer, or to verbalizing them to a non-judgmental, nonprofessional age peer. Based on prior qualitative investigations into study subjects' similar experiences of interviewer-administered assessments (Kurtz et al., 2013; Buttram \& Kurtz, 2015b), we believe that participants often perceive interviewers as concerned for their wellbeing and derive personal satisfaction or motivation to change from the positive relational aspect of the interview experience.

Certainly, it may be desirable to minimize reactive effects of assessments in many research studies. However, the overarching goal of this study was to find an acceptable, feasible and efficacious intervention approach for not-in-treatment young adults with complex patterns of multidrug use. Although an interviewer-administered assessment modality may be more difficult to scale up compared to a self-administered approach, this study nevertheless demonstrates the efficacy of a single session intervention that is acceptable to the target group. Next steps should include testing this intervention in settings where members of this population are routinely found, such as drug and DUI diversion programs, STI clinics, college campuses and social service agencies.

\subsection{Effects of study participation and assessment modality on outcome measures}

Our study findings advance the science of behavioral research among people who use drugs in a number of other areas. First and foremost, the study responds to important gaps in the literature regarding the effects of research study assessments on outcomes by including both a control group and a modality comparison in a randomized design (Kurtz, Stall, Buttram, Surratt, \& Chen, 2013; Worden \& McCrady, 2015). The inclusion of the waitlist control arm allows us to estimate, among a large sample of not-in-treatment young adults with complex multidrug use, the influences of widely recognized but rarely measured factors on substance use and sexual risk behavior outcomes: research study enrollment and participation, data collection, regression to the mean, readiness to change, and spontaneous remission. In the present study, all participants were also offered voluntary HIV counseling and testing, and uptake was high.

The effect size for reduction in drug use was in the medium range for control group participants, but there were not significant improvements in measures of abstinence, mental distress or substance dependence symptoms. Unfortunately, our study design does not enable us to tease out specific reasons for reduction in the primary drug use outcome measure among controls. Large-scale randomized studies with sufficient power to examine end-point differences with no baseline or interim data collection from the control group would be needed to distinguish the effects of even minimal data collection from other study participation influences.

In terms of the effects of assessment modality on data validity, we found no differences between the CAPI and ACASI arms on any baseline measures of demographics, health risk behaviors, or health problems. This finding is at odds with some research that shows ACASI 
to increase the reporting of sensitive and/or stigmatizing behaviors, such as drug use (Des Jarlais et al., 1999; Newman et al., 2002) and sexual behaviors (Ghanem, Hutton, Zenilman, Zimba, \& Erbelding, 2005; Gorbach et al., 2013; Kurth et al., 2004), or both (Turner et al., 1998), compared to interviewer-administered methods.

Our interpretation of this finding is that the inhibiting effects of social desirability bias on risk behavior reporting in face-to-face interviews are context-dependent. In the present study, participants were young adults with high levels of drug use and sexual risk behaviors. Based on prior work (Kurtz et al., 2013; Kurtz, 2012), we believe that not-in-treatment persons with extensive drug involvement provide valid self-reports of risk behavior in faceto-face interviews with non-judgmental interviewers because this information is not "sensitive" for them in these settings. Moreover, most of the research in the U.S. that supports the greater validity of ACASI over CAPI for collecting sensitive behavioral data is more than a decade old and included clinic-based samples. In terms of context, then, we postulate that we did not observe social desirability bias in the present study because of both participant and environmental factors: 1) young adults who participate in risky substance use and sexual behaviors with their peers may not perceive questions about sex or drug use to be stigmatizing; and 2) social desirability bias is muted in the presence of non-judgmental agepeer interviewers who do not hold positions of clinical expertise or authority.

\subsection{Limitations and strengths}

Some limitations of our study merit attention. First, while our sample was representative of the gender and racial/ethnic composition of the region, it is unclear whether the study findings generalize to not-in-treatment young adult populations who use drugs in different contexts, have lower multi-drug use, or are engaged exclusively in same-sex behaviors. The sample recruited for this study exhibited very high levels of drug involvement and other health and social problems. Second, all data are based on self-report, and did not include drug use biomarkers. Given the high levels of substance use and sexual risk behaviors we found, however, as well as the consistency of reports across assessment modalities with random assignment, underreporting of stigmatized behaviors would appear to be minimal. Third, mental health symptoms were self-identified rather than assessed by a clinician, such that caution is warranted when comparing mental health symptomology reported here with other studies. However, there is evidence that young adults are the best informants of their internalizing behaviors (Pagano, Cassidy, Murphy, \& Jellinek, 2000).

Fourth, we note that the ACASI assessment programming and setting we provided were highly structured, providing little opportunity for participants to "surf" through items and avoid meaningful responses (Ghanem et al., 2005). Finally, there is no perfect substitute for data collected directly from respondents, which were missing at baseline for secondary outcomes for control arm participants (who provided only drug use and sexual behavior frequency data at baseline). However, robust methods for multiple imputation generated baseline secondary outcome scores for controls that were comparable to the other arm conditions. Strengths of this study include a large and diverse sample of young adults with complex multidrug use, excellent balance of baseline characteristics across assigned 
conditions, high retention rates, psychometrically sound measures, and statistical techniques that produced robust parameter estimates.

\subsection{Implications for Future Research}

We believe that the present study advances the science of behavioral intervention research among substance users in important ways. We demonstrated that, for the target population, assessment modality had no apparent inhibiting effect on the reporting of "sensitive" personal data, including drug use and sexual behaviors. Second, the study established the therapeutic benefit of interviewer interaction in reducing risky behavior among this young, club drug using population.

Third, and most importantly, we demonstrated the efficacy and acceptability of a low threshold intervention in reducing drug use, sexual risk behaviors and related co-morbidities among a not-in-treatment young adult population that exhibits severe and complex levels of drug use, but that is also highly resistant to intervention. Given the limited availability of substance abuse treatment slots for this population, and the comparative cost efficiencies of the assessment approach compared to formal treatment, future studies should prioritize testing the CAPI assessment intervention in community and clinical settings.

\section{Acknowledgments}

This research was supported by the National Institute on Drug Abuse [grant number 5 R01 DA019048]. The contents are solely the responsibility of the authors and do not represent the official views of the National Institutes of Health or the National Institute on Drug Abuse.

\section{References}

Abdulrahim, D., Bowden-Jones, O. Guidance on the clinical management of acute and chronic harms of club drugs and novel psychoactive substances. London, UK: Novel Psychoactive Treatment UK Network (NEPTUNE); 2015.

Allison, P. Logistic regression using SAS: Theory and application. Cary, NC: SAS Institute; 1999.

Allison, PD. Logistic regression using SAS: Theory and application. 2nd. Cary, NC: SAS Institute; 2005.

American Psychiatric Association. American Psychiatric Association diagnostic and statistical manual of mental disorders. 4th. Washington, DC: American Psychiatric Association; 1994.

Bandura, A. Social foundations of thought and action: A social cognitive theory. Englewood Cliffs, NJ: Prentice-Hall; 1986.

Brandt PR. Journeys; South Beach: From hot to cold, back to hot again. New York Times. 2003 Dec 5.:F1.

Budney AJ, Stanger C, Tilford JM, Scherer E, Brown PC, Li Z, Li Z, Walker D. Computer-assisted Behavioral Therapy and Contingency Management for Cannabis Use Disorder. Psychology of Addictive Behaviors. 2015; 29(3):501-511. [PubMed: 25938629]

Buttram ME, Kurtz SP. Characteristics associated with group sex participation among men and women in the club drug scene. Sexual Health. 2015a; 12(6):560-562. [PubMed: 26342286]

Buttram ME, Kurtz SP. A qualitative study of African American/Black MSM's experiences of participating in a substance use and sexual risk reduction intervention. American Journal of Men's Health. 2015b; doi: 10.1177/1557988315584157

Buttram ME, Kurtz SP. Alternate routes of administration among prescription opioid misusers and associations with sexual HIV transmission risk Behaviors. Journal of Psychoactive Drugs. 2016; 48(3):187-194. [PubMed: 27224253] 
Carlson RG, Falck RS, McCaughan JA, Siegal HA. MDMA/Ecstasy use among young people in Ohio: Perceived risk and barriers to intervention. Journal of Psychoactive Drugs. 2004; 36(2):181-189. [PubMed: 15369199]

Chinet L, Stephan P, Zobel F, Halfon O. Party drug use in techno nights: A field survey among Frenchspeaking Swiss attendees. Pharmacology Biochemistry and Behavior. 2007; 86(2):284-289.

Clifford PR, Maisto SA. Subject reactivity effects and alcohol treatment outcome research. Journal of Studies on Alcohol. 2000; 61(6):787-793. [PubMed: 11188483]

Clifford PR, Maisto SA, Davis CM. Alcohol treatment research assessment exposeure subject reactivity effects: Part I. Alcohol use and related consequences. Journal of Studies on Alcohol and Drugs. 2007; 68(4):519-528. [PubMed: 17568955]

Cohen, J. Statistics power analysis for the behavioral sciences. Mahwah, NJ: Lawrence Erlbaum Associates; 1988.

Cottler LB, Womack SB, Compton WM, Ben-Abdallah A. Ecstasy abuse and dependence among adolescents and young adults: Applicability and reliability of DSM-IV criteria. Human Psychopharmacology: Clinical and Experimental. 2001; 16:599-606. [PubMed: 12404539]

Dennis, ML. Global Appraisal of Individual Needs Version 5.4. Bloomington, IL: Chestnut Health Systems; 2006.

Dennis, ML., Rourke, KM., Lennox, R., Campbell, RS., Caddell, JM. Global Appraisal of Individual Needs: Background and Psychometric Properties Manual (GAIN-B). Research Triangle Park, NC: Research Triangle Institute; 1995.

Des Jarlais D, Paone D, Milliken J, Turner CF, Miller H, Gribble J, ... Friedman SR. Audio-computer interviewing to measure risk behaviour for HIV among injecting drug users: a quasi-randomised trial. Lancet. 1999; 353

Epstein EE, Drapkin ML, Yusko DA, Cook SM, McCrady BS, Jensen NK. Is alcohol assessment therapeutic? Pretreatment change in drinking among alcohol-dependent women. Journal of Studies on Alcohol. 2005; 66(3):369-378. [PubMed: 16047526]

Falck RS, Carlson RG, Wang J, Siegal HA. Sources of information about MDMA (3,4methylenedioxymethamphetamine): Perceived accuracy, importance, and implications for prevention among young adult users. Drug and Alcohol Dependence. 2004; 74:45-54. [PubMed: 15072806]

German CL, Fleckenstein AE, Hanson GR. Bath salts and synthetic cathinones: An emerging designer drug phenomenon. Life Sciences. 2014; 97(1):2-8. [PubMed: 23911668]

Ghanem KG, Hutton HE, Zenilman JM, Zimba R, Erbelding EJ. Audio computer assisted self interview and face to face interview modes in assessing response bias among STD clinic patients. Sexually Transmitted Infectections. 2005; 8(1):421-425.

Gorbach PM, Mensch BS, Husnik M, Coly A, Mâsse B, Makanani B, ... Forsyth A. Effect of computer-assisted interviewing on self-reported sexual behavior data in a microbicide clinical trial. AIDS and Behavior. 2013; 17(2):790-800. [PubMed: 23054034]

Greist JH, Marks IM, Baer L, Kobak KA, Wenzel KW, Hirsch MJ, ... Clary CM. Behavior therapy for obsessive-compulsive disorder guided by a computer or by a clinician compared with relaxation as a control. Journal of Clinical Psychiatry. 2002; 63(2):138-145. [PubMed: 11874215]

Heckathorn DD. Respondent-Driven Sampling: A New Approach to the Study of Hidden Populations. Social Problems. 1997; 44(2):174-199.

Heckathorn DD. Respondent-Driven Sampling II: Deriving Valid Population Estimates from ChainReferral Samples of Hidden Populations. Social Problems. 2002; 49(1):11-34.

Kelly BC, Parsons JT. Prescription drug misuse among club drug-using young adults. The American Journal of Drug and Alcohol Abuse. 2007; 33(6):875-884. [PubMed: 17994483]

Kelly BC, Wells BE, Pawson M, LeClair A, Parsons JT. Combinations of prescription drug misuse and illicit drugs among young adults. Addictive Behaviors. 2014; 39(5):941-944. [PubMed: 24462348]

Kurth AE, Martin DP, Golden MR, Weiss NS, Heagerty PJ, Spielberg F, ... Holmes KK. A Comparison Between Audio Computer-Assisted Self-Interviews and Clinician Interviews for Obtaining the Sexual History. Sexually Transmitted Diseases. 2004; 31(12):719-726. [PubMed: 15608586] 
Kurtz, SP. Arrest histories, victimization, substance use, and sexual risk behaviors among young adults in Miami's club scene. In: Sanders, B.Thomas, YF., Deeds, B., editors. Crime, HIV, and Health: Intersections of Criminal Justice and Public Health Concerns. New Yorks, NY: Springer; 2012. p. 151-166.

Kurtz SP, Inciardi J, Pujals E. Criminal activity among young adults in the club scene. Law Enforcement Executive Forum. 2009; 9(2):47-59. [PubMed: 20390051]

Kurtz SP, Inciardi JA, Surratt HL, Cottler L. Prescription drug abuse among ecstasy users in Miami. Journal of Addictive Diseases. 2005; 24(4):1-16.

Kurtz SP, Stall RD, Buttram ME, Surratt HL, Chen M. A Randomized Trial of a Behavioral Intervention for High Risk Substance-Using MSM. AIDS and Behavior. 2013; 17(29):2914-2926. DOI: 10.1007/s10461-013-0531-z [PubMed: 23732957]

Kurtz SP, Surratt HL, Buttram ME, Levi-Minzi MA, Chen M. Interview as Intervention: The Case of Young Adult Multidrug Users in the Club Scene. Journal of Substance Abuse Treatment. 2013; 44(3):301-308. [PubMed: 22971689]

Kurtz SP, Surratt HL, Levi-Minzi MA, Mooss A. Benzodiazepine dependence among multidrug users in the club scene. Drug and Alcohol Dependence. 2011; 119(1):99-105. [PubMed: 21708434]

Lightfoot M, Comulada SW, Stover G. Computerized HIV preventive intervention for adolescents: Indications for efficacy. American Journal of Public Health. 2007; 97(6):1027-1030. [PubMed: 16670219]

Little, RJA., Rubin, DB. Statistical analysis with missing data. Second Edition. New York: John Wiley; 2002.

Marsden J, Stillwell G, Barlow H, Boys A, Taylor C, Hunt N, Farrell M. An evaluation of a brief motivational intervention among young ecstasy and cocaine users: No effect on substance and alcohol use outcomes. Addiction. 2006; 101:1014-1026. [PubMed: 16771893]

McKendree-Smith NL, Floyd M, Scogin FR. Self-administered treatments for depression: A review. Journal of Clinical Psychology. 2003; 59(3):275-288. [PubMed: 12579545]

Mitcheson L, McCambridge J, Byrne A, Hunt N, Winstock A. Sexual health risk among dance drug users: Cross-sectional comparisons with nationally representative data. International Journal of Drug Policy. 2008; 19(4):304-310. [PubMed: 18638703]

Musselman ME, Hampton JE. Not for human consumption: A review of emerging designer Drugs. Pharmacotherapy: The Journal of Human Pharmacology and Drug Therapy. 2014; 34(7):745-757.

National Institute on Drug Abuse 2009. [Accessed December 12, 2016] Club Drugs. Jul 27. 2009 Retrieved from http://www.nida.nih.gov/DrugPages/Clubdrugs.html

Newman JC, Des Jarlais DC, Turner CF, Gribble J, Cooley P, Paone D. The differential effects of faceto-face and computer interview modes. American Journal of Public Health. 2002; 92(2):294-297. [PubMed: 11818309]

Newman MG. Technology in psychotherapy: An introduction. Journal of Clinical Psychology. 2004; 60(2):141-145. [PubMed: 14724921]

Nicholi AM Jr. The nontherapeutic use of psychoactive drugs: A modern epidemic. New England Journal of Medicine. 1983; 308(16):925-933. [PubMed: 6339924]

Noar SM, Black HG, Pierce LB. Efficacy of computer technology-based HIV prevention interventions: A meta-analysis. AIDS. 2009; 23:107-115. [PubMed: 19050392]

Norberg MN, Hides L, Olivier J, Khawar L, McKetin R, Copeland J. Brief interventions to reduce ecstasy use: a multi-site randomised controlled trial. Behavior Therapy. 2014; 45:745-759. [PubMed: 25311285]

Novoa RA, Ompad DC, Wu Y, Vlahov D, Galea S. Ecstasy use and its association with sexual behaviors among drug users in New York City. Journal of Community Health. 2005; 30(5):331343. [PubMed: 16175956]

Owen, F. Clubland: The Fabulous Rise and Murderous Fall of Club Culture. New York: St: Martin's Press; 2003.

Pagano ME, Cassidy LJ, Murphy LM, Jellinek MS. Identifying psychosocial dysfunction in school-age children: The pediatric symptom checklist as a self-report measure. Psychology in the Schools. 2000; 37(2):91-106. [PubMed: 22328794] 
Pagano ME, Zemore SE, Onder CC, Stout RL. Predictors of initial AA-related helping: Findings from Project MATCH. Journal of Studies on Alcohol and Drugs. 2009; 70(1):117-125. [PubMed: 19118400]

Parrott AC, Milani RM, Parmar R, Turner JJD. Recreational ecstasy/MDMA and other drug users from the UK and Italy: Psychiatric symptoms and psychobiological problems. Psychopharmacology. 2001; 159:77-82. [PubMed: 11797073]

Parsons JT, Grov C, Kelly BC. Club drug use and dependence among young adults recruited through time-space sampling. Public Health Reports. 2009; 124(2):246-254. [PubMed: 19320366]

Perrone, D. The high life: Club kids, harm and drug policy. Monsey, NY: Crinimal Justice Press; 2009.

Reynolds, S. Generation Ecstasy: Into the World of Techno and Rave Culture. Boston: Little, Brown and Company; 1998.

Saitz R. Screening and brief intervention for unhealthy drug use: Little or no efficacy. Frontiers in Psychiatry. 2014; 5

Schaffer A. Online treatment may help insomniacs. The New York Times. 2009 Aug 11.:D9.

Schifano F, Di Furia L, Gorza G, Minicuci N, Bricolo R. MDMA ('ecstasy') consumption in the context of polydrug abuse: A report on 150 patients. Drug and Alcohol Dependence. 1998; 52(1): 85-90. [PubMed: 9788011]

Schulz KF, Altman DG, Moher D. for the CONSORT Group. CONSORT 2010 statement: updated guidelines for reporting parallel group randomized trials. PLOS Medicine. 2010; 7(3):1-6.

Silcott, M. Rave America: New school dancescapes. Toronto, Ontario, Canada: ECW Press; 1999.

Sillins E, Bleeker AM, Simpson M, Dillon P, Copeland J. Does peer-delivered information at music events reduce ecstasy and methamphetamine use at three month follow-up? Findings from a quasiexperiment across three study sites. Journal of Addiction Prevention. 2013; 1(3):8.

Singer LT, Linares TJ, Ntiri S, Henry R, Minnes S. Psychosocial profiles of older adolescent MDMA users. Drug and Alcohol Dependence. 2004; 74(3):242-252.

Sterk CE, Klein H, Elifson KW. Young adult ecstasy users and multiple sexual partners: Understanding the factors underlying this HIV risk practice. Journal of Psychoactive Drugs. 2008; 40(3):237-244. [PubMed: 19004415]

Turner CF, Ku L, Rogers SM, Lindberg LD, Pleck JH, Sonenstein FL. Adolescent sexual behavior, drug use, and violence: Increased reporting with computer survey technology. Science. 1998; 8(280):867-873.

U.S. Census Bureau. [Accessed December 12, 2016] State and County Quickfacts, Miami-Dade County, Florida. 2015. Retrieved from http://www.census.gov/quickfacts/table/PST045215/12086

Voas RB, Johnson MB, Miller BA. Alcohol and drug use among young adults driving to a drinking location. Drug and Alcohol Dependence. 2013; 132(1):69-73. [PubMed: 23415848]

White HR, Jiao Y, Ray AE, Huh D, Atkins DC, Larimer ME, ... Mun E. Are there secondary effects on marijuana use from brief alcohol interventions for college students? Journal of Studies on Alcohol and Drugs. 2015; 76(3):367-377. [PubMed: 25978822]

Whittingham JRD, Ruiter RAC, Bolier L, Lemmers L, Van Hasset N, Kok G. Avoiding counterproductive results: An experimental pretest of a harm reduction intervention on attitude toward party drugs among users and nonusers. Substance Use \& Misuse. 2009; 44(4):532-547. [PubMed: 19242864]

Worden BL, McCrady BS. Pre-treatment assessment-related reductions in drinking among women with alcohol use disorders. Substance Use \& Misuse. 2015; 50(2):215-225. [PubMed: 25295598]

Young MM, Stevens A, Galipeau J, Pirie T, Garritty C, Singh K, ... Moher D. Effectiveness of brief interventions as part of the Screening, Brief Interventiona nd Referral to Treatment (SBIRT) model for reducing the nonmedical use of psychoactive substances: A systematic review. Systematic Reviews. 2014; 3(1):1. [PubMed: 24383422] 


\section{Highlights}

- Young adults in the club scene have intense and complex patterns of multidrug use.

- Assessments are effective interventions for young adults who use drugs.

- We found no social desirability bias differences in CAPI and ACASI modalities.

- The study found a therapeutic benefit of interviewer interaction. 


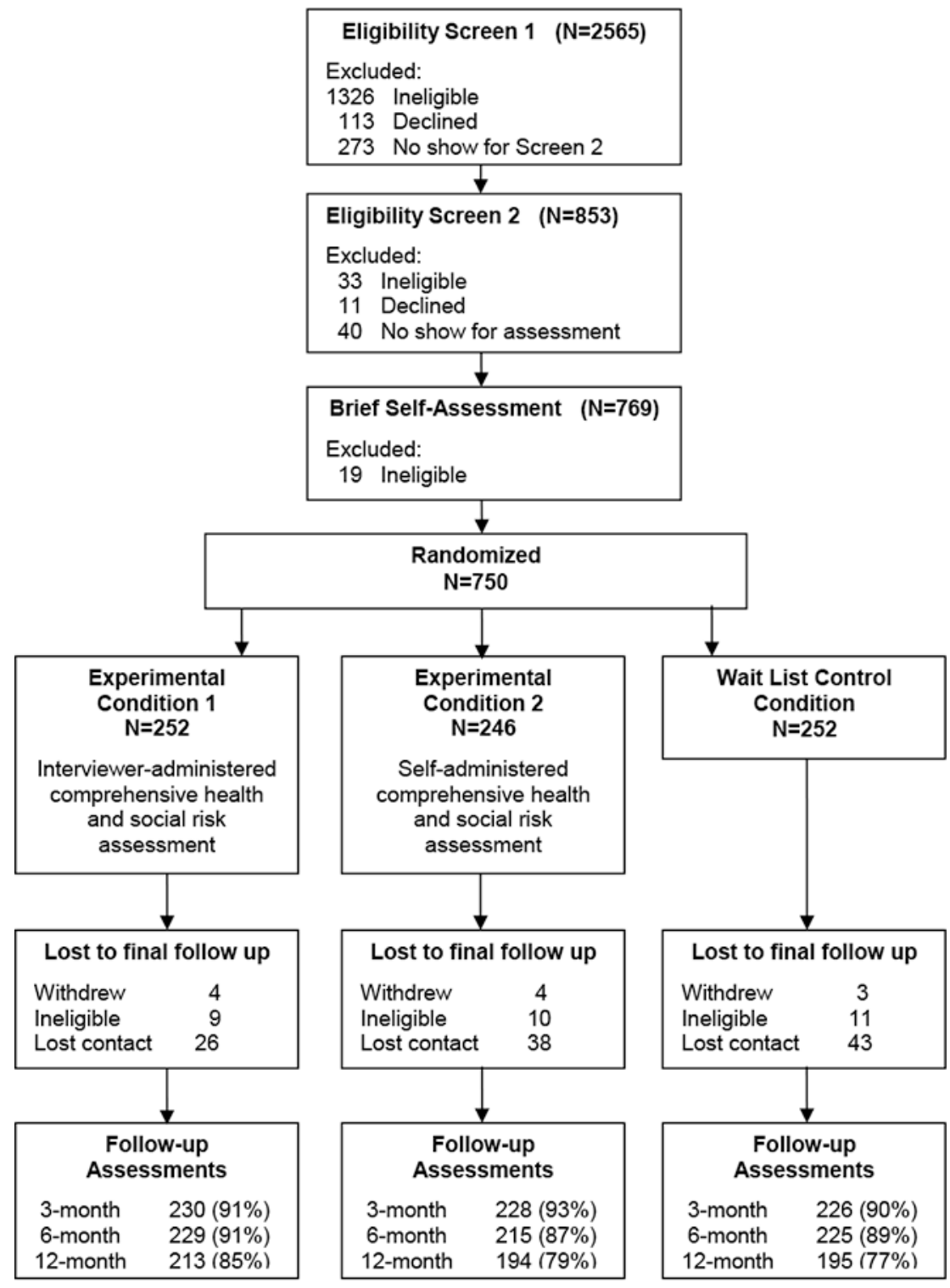

Figure I. Study Flow Chart 


\section{을 \\ 골}

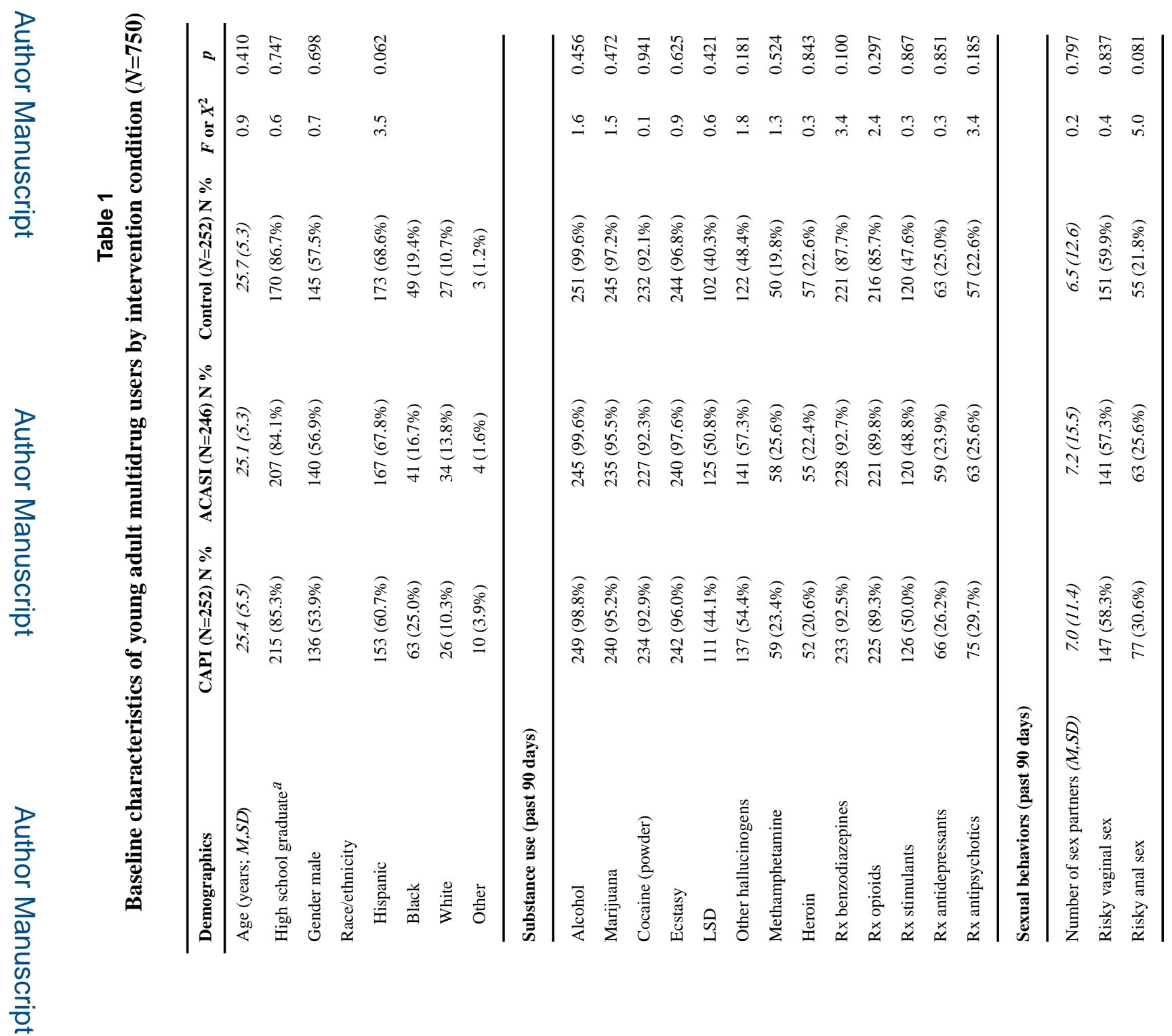

J Subst Abuse Treat. Author manuscript; available in PMC 2018 July 01. 
Kurtz et al.

Page 21

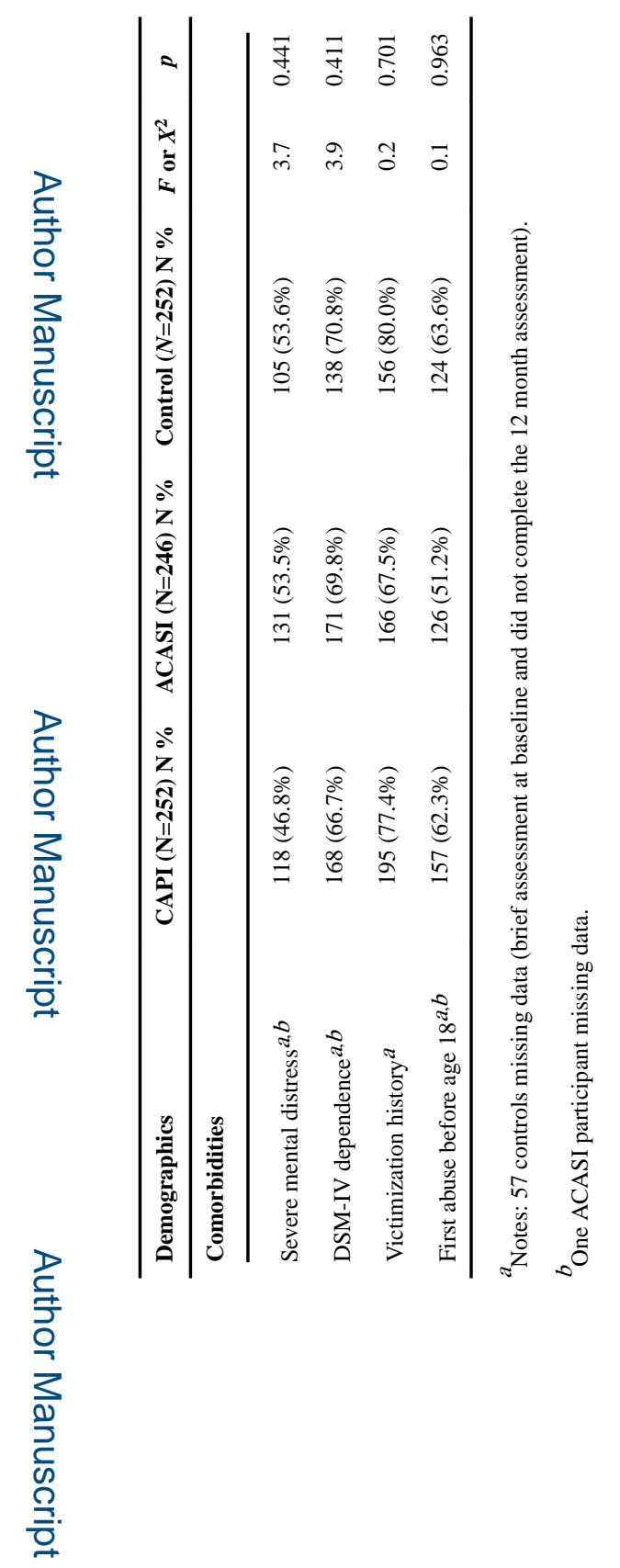

로을

J Subst Abuse Treat. Author manuscript; available in PMC 2018 July 01. 


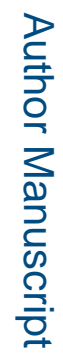

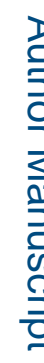

\section{$\frac{\sqrt{2}}{3}$}
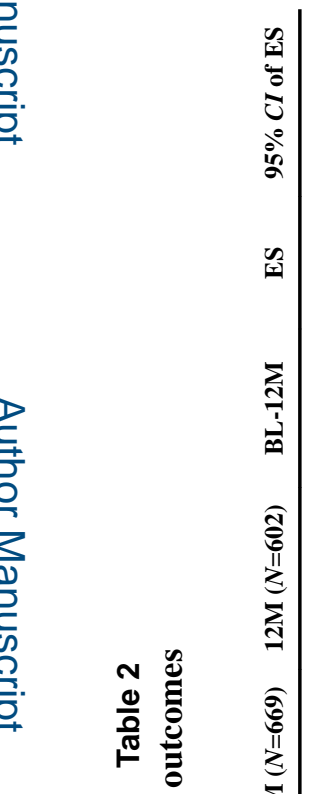

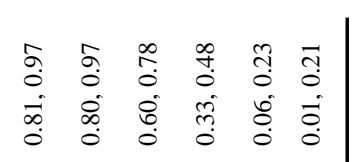

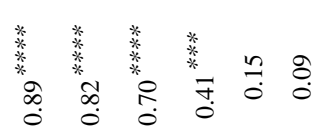

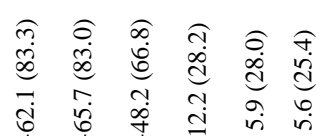

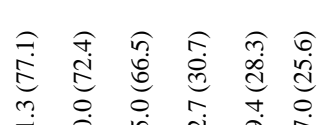

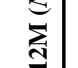

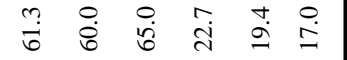

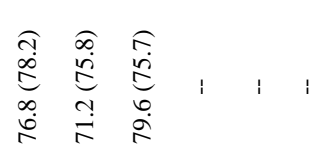

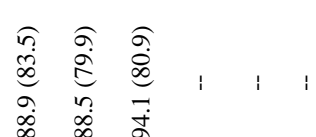

$\infty \begin{array}{lll}\infty & \infty & 0 \\ \infty & 0\end{array}$

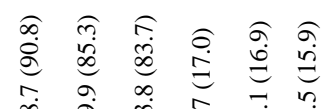

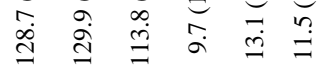

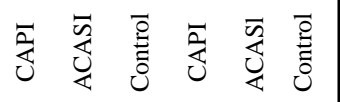

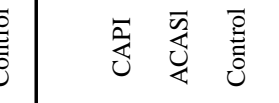

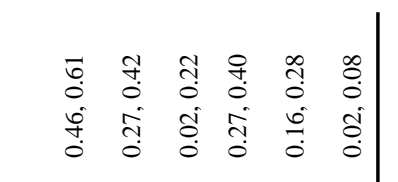

กิ =

*

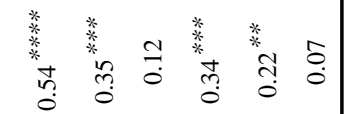

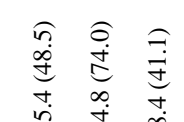

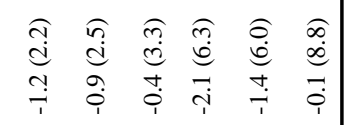

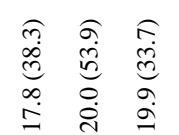

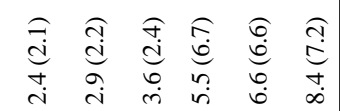

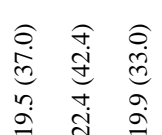

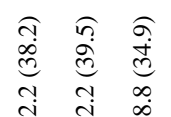

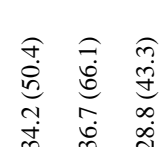

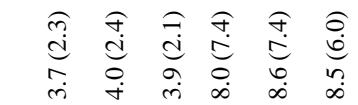

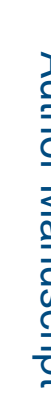

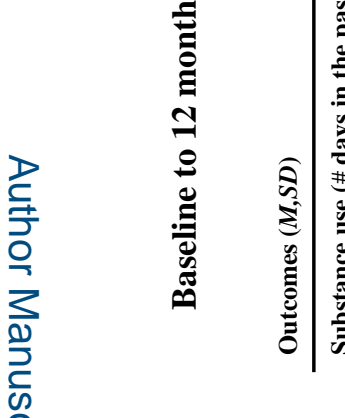
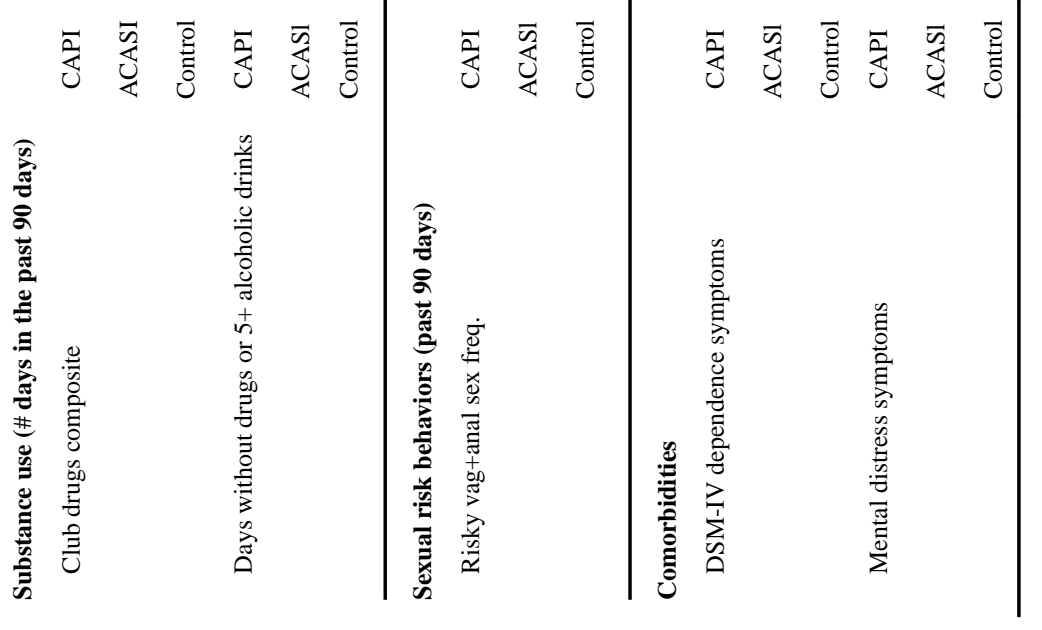

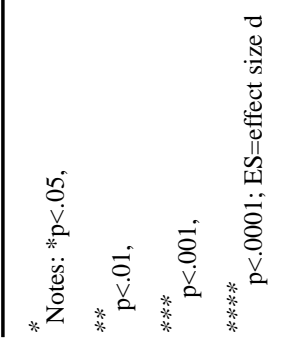

J Subst Abuse Treat. Author manuscript; available in PMC 2018 July 01. 


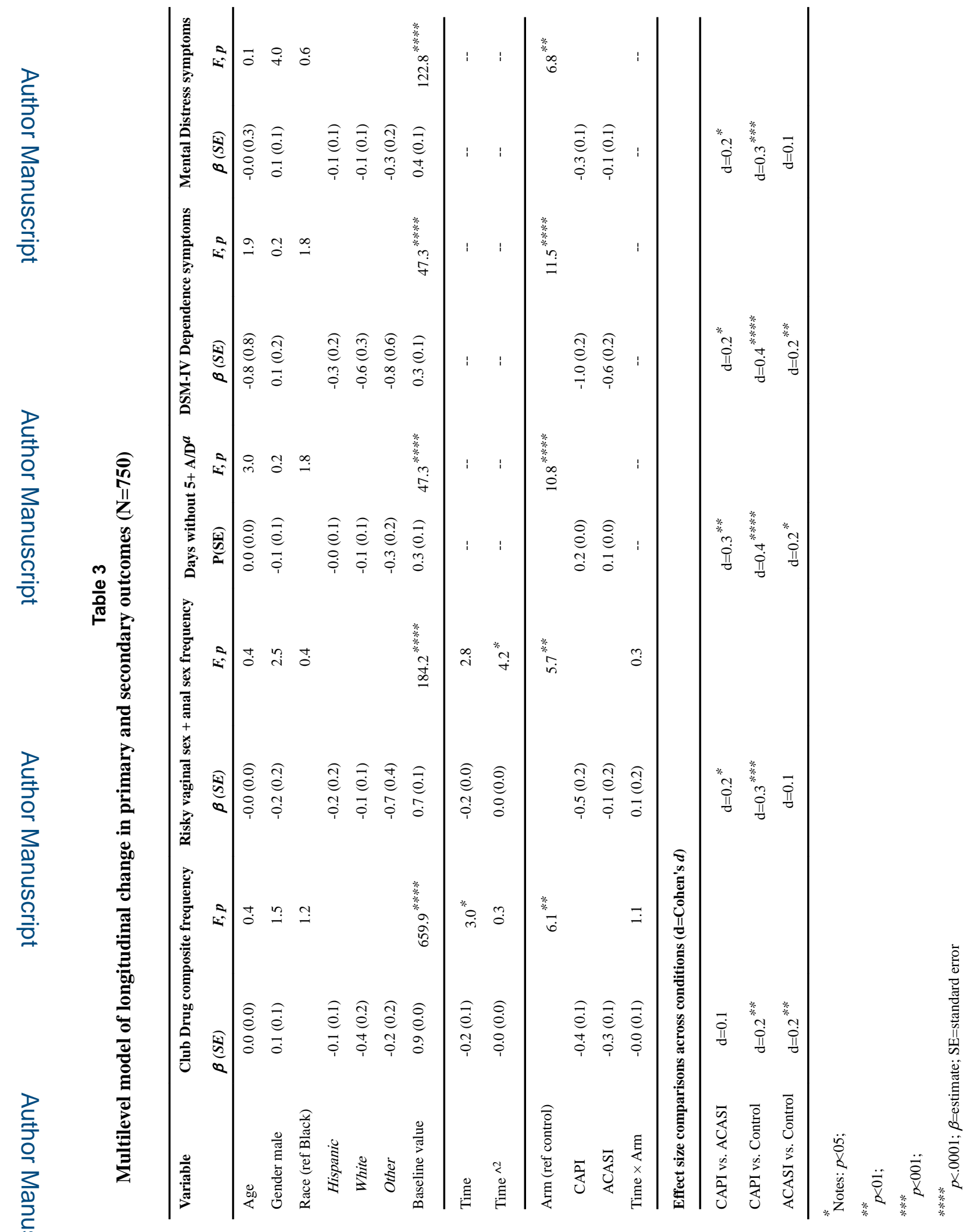

J Subst Abuse Treat. Author manuscript; available in PMC 2018 July 01. 